\title{
INTEGRAL Detection of the First Prompt Gamma-Ray Signal Coincident with the Gravitational-wave Event GW170817
}

\section{Savchenko, V.; Ferrigno, C.; Kuulkers, E.; Bazzano, A.; Bozzo, E.; Brandt, S.; Chenevez, J.; Courvoisier,} T. J.-L.; Diehl, R.; Domingo, A.

Total number of authors:

23

Published in:

The Astrophysical Journal Letters

Link to article, DOI:

10.3847/2041-8213/aa8f94

Publication date:

2017

Document Version

Publisher's PDF, also known as Version of record

Link back to DTU Orbit

Citation (APA):

Savchenko, V., Ferrigno, C., Kuulkers, E., Bazzano, A., Bozzo, E., Brandt, S., Chenevez, J., Courvoisier, T. J-L., Diehl, R., Domingo, A., Hanlon, L., Jourdain, E., von Kienlin, A., Laurent, P., Lebrun, F., Lutovinov, A., MartinCarrillo, A., Mereghetti, S., Natalucci, L., ... Úbertini, P. (2017). INTEGRAL Detection of the First Prompt Gamma-Ray Signal Coincident with the Gravitational-wave Event GW170817. The Astrophysical Journal Letters, 848(2), [L15]. https://doi.org/10.3847/2041-8213/aa8f94

\section{General rights}

Copyright and moral rights for the publications made accessible in the public portal are retained by the authors and/or other copyright owners and it is a condition of accessing publications that users recognise and abide by the legal requirements associated with these rights.

- Users may download and print one copy of any publication from the public portal for the purpose of private study or research.

- You may not further distribute the material or use it for any profit-making activity or commercial gain

- You may freely distribute the URL identifying the publication in the public portal 


\title{
INTEGRAL Detection of the First Prompt Gamma-Ray Signal Coincident with the Gravitational-wave Event GW170817
}

\author{
V. Savchenko ${ }^{1}$ (D) C. Ferrigno ${ }^{1}$ (D) , E. Kuulkers ${ }^{2}$, A. Bazzano ${ }^{3}$ (D), E. Bozzo ${ }^{1}$ (D), S. Brandt ${ }^{4}$ J. Chenevez $^{4}$ (D), T. J.-L. Courvoisier ${ }^{1}$ (D), \\ R. Diehl ${ }^{5}$, A. Domingo ${ }^{6}$, L. Hanlon ${ }^{7}$ (D), E. Jourdain ${ }^{8}$ (D), A. von Kienlin ${ }^{5}$ (D), P. Laurent ${ }^{9,10}$ (iD), F. Lebrun ${ }^{9}$, A. Lutovinov ${ }^{11,12}$ (D), \\ A. Martin-Carrillo ${ }^{7}$, S. Mereghetti ${ }^{13}$ (D) L. Natalucci ${ }^{3}$, J. Rodi $^{3}$, J.-P. Roques ${ }^{8}$, R. Sunyaev ${ }^{11,14}$, and P. Ubertini ${ }^{3}$ \\ ${ }_{1}^{1}$ ISDC, Department of Astronomy, University of Geneva, Chemin d'Écogia, 16 CH-1290 Versoix, Switzerland \\ ${ }^{2}$ European Space Research and Technology Centre (ESA/ESTEC), Keplerlaan 1, 2201 AZ Noordwijk, The Netherlands \\ ${ }^{3}$ INAF-Institute for Space Astrophysics and Planetology, Via Fosso del Cavaliere 100, I-00133-Rome, Italy \\ ${ }^{4}$ DTU Space, National Space Institute Elektrovej, Building 327 DK-2800 Kongens Lyngby, Denmark \\ ${ }^{5}$ Max-Planck-Institut für Extraterrestrische Physik, Garching, Germany \\ ${ }^{6}$ Centro de Astrobiología (CAB-CSIC/INTA, ESAC Campus), Camino bajo del Castillo S/N, E-28692 Villanueva de la Cañada, Madrid, Spain \\ ${ }^{7}$ Space Science Group, School of Physics, University College Dublin, Belfield, Dublin 4, Ireland \\ 8 IRAP, Université de Toulouse, CNRS, UPS, CNES; 9 Av. Roche, F-31028 Toulouse, France \\ ${ }^{9}$ APC, AstroParticule et Cosmologie, Université Paris Diderot, CNRS/IN2P3, CEA/Irfu, Observatoire de Paris Sorbonne Paris Cité, \\ 10 rue Alice Domont et Léonie Duquet, F-75205 Paris Cedex 13, France \\ ${ }^{10}$ DSM/Irfu/Service d'Astrophysique, Bat. 709 Orme des Merisiers CEA Saclay, F-91191 Gif-sur-Yvette Cedex, France \\ ${ }^{11}$ Space Research Institute of Russian Academy of Sciences, Profsoyuznaya 84/32, 117997 Moscow, Russia \\ ${ }^{12}$ Moscow Institute of Physics and Technology, Institutskiy per. 9, 141700 Dolgoprudny, Moscow Region, Russia \\ ${ }^{13}$ INAF, IASF-Milano, via E.Bassini 15, I-20133 Milano, Italy \\ ${ }^{14}$ Max Planck Institute for Astrophysics, Karl-Schwarzschild-Str. 1, D-85741 Garching b. München, Germany \\ Received 2017 September 19; revised 2017 September 27; accepted 2017 September 27; published 2017 October 16
}

\begin{abstract}
We report the INTernational Gamma-ray Astrophysics Laboratory (INTEGRAL) detection of the short gamma-ray burst GRB 170817A (discovered by Fermi-GBM) with a signal-to-noise ratio of 4.6, and, for the first time, its association with the gravitational waves (GWs) from binary neutron star (BNS) merging event GW170817 detected by the LIGO and Virgo observatories. The significance of association between the gamma-ray burst observed by INTEGRAL and GW170817 is $3.2 \sigma$, while the association between the Fermi-GBM and INTEGRAL detections is $4.2 \sigma$. GRB 170817A was detected by the SPI-ACS instrument about $2 \mathrm{~s}$ after the end of the GW event. We measure a fluence of $(1.4 \pm 0.4 \pm 0.6) \times 10^{-7} \mathrm{erg} \mathrm{cm}^{-2}(75-2000 \mathrm{keV})$, where, respectively, the statistical error is given at the $1 \sigma$ confidence level, and the systematic error corresponds to the uncertainty in the spectral model and instrument response. We also report on the pointed follow-up observations carried out by INTEGRAL, starting $19.5 \mathrm{hr}$ after the event, and lasting for 5.4 days. We provide a stringent upper limit on any electromagnetic signal in a very broad energy range, from $3 \mathrm{keV}$ to $8 \mathrm{MeV}$, constraining the soft gamma-ray afterglow flux to $<7.1 \times 10^{-11} \mathrm{erg} \mathrm{cm}^{-2} \mathrm{~s}^{-1}(80-300 \mathrm{keV})$. Exploiting the unique capabilities of INTEGRAL, we constrained the gamma-ray line emission from radioactive decays that are expected to be the principal source of the energy behind a kilonova event following a BNS coalescence. Finally, we put a stringent upper limit on any delayed bursting activity, for example, from a newly formed magnetar.
\end{abstract}

Key words: gamma rays: general - gamma-ray burst: general - gravitational waves

\section{Introduction}

It has long been conjectured that the subclass of gamma-ray bursts (GRBs) with a duration below about $2 \mathrm{~s}$, known as short gamma-ray bursts (sGRBs), are the product of a binary neutron star (BNS) merger and that gamma-rays are produced in the collimated ejecta following the coalescence (e.g., Blinnikov et al. 1984; Nakar 2007; Gehrels \& Meszaros 2012; Berger 2014). So far, there was only circumstantial evidence for this hypothesis, owing to the lack of supernovae associated with sGRBs, their localization in early-type galaxies, and their distinct class of duration (e.g., D'Avanzo 2015). The advent of advanced gravitational-wave (GW) detectors, which have been able to detect binary black hole mergers (Abbott et al. 2016a, 2016b, 2016c, 2017; LIGO Scientific Collaboration \& Virgo Collaboration 2017a) and have the capability to detect a signal from nearby BNS mergers (Abbott et al. 2016c), have sparked great expectations. Different electromagnetic signatures are expected to be associated with BNS merger events, owing to expanding ejecta, the most obvious of which is an sGRB in temporal coincidence with the GW signal and/or afterglow emission at different wavelengths in the days and/or weeks after the merger event (e.g., Fernández \& Metzger 2016).

On 2017 August 17 at 12:41:04.47 UTC ( $T_{0, \mathrm{GW}}$ hereafter), a signal consistent with the merger of a BNS was detected by the LIGO-Hanford detector (LIGO Scientific Collaboration et al. 2015) as a single-detector trigger. The subsequent alert was issued in response to a public real-time Fermi Gamma-ray Burst Monitor (GBM) trigger on an sGRB at 12:41:06.48 UTC (Connaughton 2017; LIGO Scientific Collaboration \& Virgo Collaboration 2017a, 2017d); the GRB signal was immediately and independently confirmed by our team (Savchenko et al. 2017b).

Analysis of the LIGO-Livingston data (LIGO Scientific Collaboration \& Virgo Collaboration 2017e) revealed that a trigger was not automatically issued due to the proximity of an overflow instrumental transient, which could be safely removed offline. The addition of Virgo (Acernese et al. 2015) to the detector network allowed a precise localization at 90\% confidence level in an area of about 31 square degrees (LIGO 
Scientific Collaboration \& Virgo Collaboration 2017b), which is consistent with the Fermi-GBM localization of GRB 170817A (von Kienlin et al. 2017). The most accurate localization so far has been derived by the LALInferrence pipeline (LIGO Scientific Collaboration \& Virgo Collaboration 2017c); this is the localization we use in this Letter.

A massive follow-up campaign of the LIGO-Virgo highprobability region by optical robotic telescopes started immediately after the event and on 2017 August 18 between 1:05 and 1:45 UT; three groups reported independent detections of a transient optical source at about 10 arcsec from the center of the host S0 Galaxy NGC 4993; this source was dubbed SSS17a (Coulter et al. 2017; D. A. Coulter et al. 2017, in preparation) or DLT17ck (Valenti et al. 2017; Yang et al. 2017); the transient source was confirmed by DESGW +Community Team (2017; see also Soares-Santos et al. 2017). The source was identified as the most probable optical counterpart of the BNS merger (Foley 2017; Siebert et al. 2017). After that, it was followed at all wavelengths. The counterpart has been given the official IAU designation “AT 2017gfo" (LIGO Scientific Collaboration et al. 2017, in preparation).

During early observations by the Swift satellite from 53.8 to $55.8 \mathrm{ks}$ after the LVC trigger an ultraviolet (UV) transient with $u$ magnitude 17 was detected; an X-ray upper limit was set at an order of magnitude below the typical luminosity of an sGRB afterglow, as determined from the sample of Swift Burst Alert Telescope (BAT) triggered objects. It was suggested that the object may be a blue (i.e., lanthanide-free) kilonova (Evans et al. 2017). Infrared spectroscopy with X-shooter on the ESO Very Large Telescope UT 2 covered the wavelength range 3000-25000 A and started roughly 1.5 days after the GW event (D'Elia et al. 2017). As reported in E. Pian et al. (2017, in preparation), strong evidence was found for r-process nucleosynthesis as predicted by kilonova models (e.g., Tanaka et al. 2017). Gemini spectroscopic observations with the Flamingos-2 instrument taken 3.5 days after the GW event revealed a red featureless spectrum, again consistent with kilonova expectations (Troja et al. 2017a, 2017b). Optical spectra collected 1.5 days after the GW event with the ESO New Technology Telescope at La Silla equipped with the EFOSC2 instrument in spectroscopic mode excluded a supernova as being the origin of the transient emission (Lyman et al. 2017). Thus, the properties of the source are fully consistent with the kilonova scenario (see Metzger 2017 for a review). A kilonova is primarily powered by the radioactive decay of elements synthesized in the outflow, which produce gamma-ray lines. These may also be directly detectable in the gamma-ray range (Hotokezaka et al. 2016).

In this Letter, we describe in detail the detection of GRB 170817A by the INTernational Gamma-ray Astrophysics Laboratory (INTEGRAL) and the targeted follow-up observing campaign. We were able to search for any possible hard X-ray/ soft gamma-ray emission for about six days after the prompt gamma-ray and GW signal. This allowed us to constrain both continuum emission from GRB-like afterglow emission and line emissions expected from kilonovae.

\section{INTEGRAL Instrument Summary}

INTEGRAL (Winkler et al. 2003) is an observatory with multiple instruments: a gamma-ray spectrometer $(20 \mathrm{keV}-8 \mathrm{MeV}$, SPI; Vedrenne et al. 2003), an imager (15 keV-10 MeV, IBIS; Ubertini et al. 2003), an X-ray monitor (3-35 keV, JEM-X; Lund

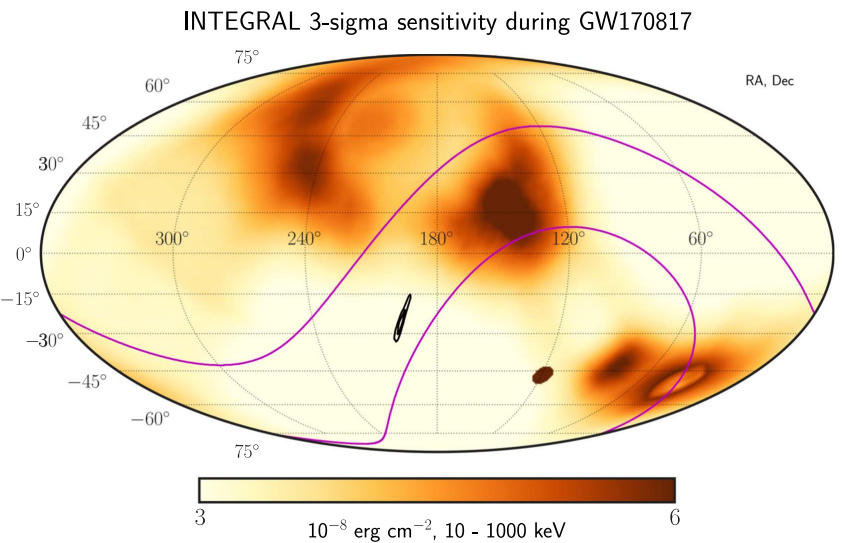

Figure 1. INTEGRAL $3 \sigma$ sensitivity to a $100 \mathrm{~ms}$ burst characterized by Comptonized emission with $\alpha=-0.65$ and $E_{\text {peak }}=185 \mathrm{keV}$, i.e., the best-fit spectral model reported by the Fermi-GBM for the pulse of the GRB. Black contours correspond to the confidence regions (90\% and 50\%) of the current LALInferrence LIGO/Virgo localization (LIGO Scientific Collaboration \& Virgo Collaboration 2017c). The magenta annulus corresponds to the constraint on the GRB 170817A location derived from the difference in arrival time of the event to Fermi and INTEGRAL (triangulation; Svinkin \& Hurley 2017).

et al. 2003), and an optical monitor (V band, Optical Monitoring Camera (OMC); Mas-Hesse et al. 2003).

The spectrometer SPI is surrounded by a thick AntiCoincidence Shield (SPI-ACS). In addition to its main function of providing a veto signal for charged particles irradiating the SPI instrument, the ACS is also able to register all other impinging particles and high-energy photons. Thus, it can be used as a nearly omnidirectional detector of transient events with an effective area reaching $0.7 \mathrm{~m}^{2}$ at energies above $\sim 75 \mathrm{keV}$ and a time resolution of $50 \mathrm{~ms}$ (von Kienlin et al. 2003). The characterization of its response to a gamma-ray signal has been delivered with an extensive simulation study, taking into account the complex opacity pattern of materials, which are used for the INTEGRAL satellite structure and other instrument detectors. Similarly, we have computed and verified the response of the other omnidirectional detectors on board INTEGRAL: IBIS/ISGRI, IBIS/PICsIT, and IBIS/Veto. For details on the INTEGRAL capabilities of detecting transients from the whole sky, particularly as relevant to our search for electromagnetic counterparts to GW signals, we refer to Savchenko et al. (2017a and references therein).

\section{Observation of the Prompt Gamma-Ray Emission}

At the time of the GW170817 trigger, INTEGRAL was performing a target-of-opportunity observation of GW170814 (Abbott et al. 2017b; LIGO Scientific Collaboration \& Virgo Collaboration 2017e) and at 12:41 UTC it was directed to R.A., decl. $(\mathrm{J} 2000.0)=36^{\circ} .25,-49^{\circ} .80$. This orientation was overall favorable to detect a signal from the location of AT 2017gfo with the SPI-ACS, although not the most optimal. This can be seen in Figure 1, where we show the complete INTEGRAL sensitivity map combining all instruments as described in Savchenko et al. (2017a). We note that with this orientation, the sensitivity of IBIS (ISGRI, Lebrun et al. 2003; PICsIT, Labanti et al. 2003; Veto, Quadrini et al. 2003 detectors) to a signal from the direction of AT 2017gfo was much lower if compared to SPI-ACS for any plausible type of event spectrum. 


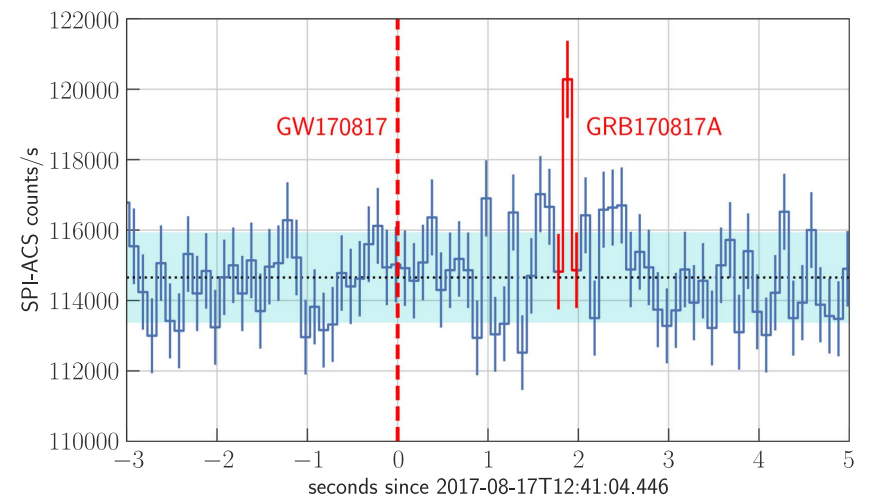

Figure 2. SPI-ACS light curve of GRB 170817A (100 ms time resolution), detected $2 \mathrm{~s}$ after GW170817. The red line highlights the $100 \mathrm{~ms}$ pulse, which has an $\mathrm{S} / \mathrm{N}$ of 4.6 in SPI-ACS. The blue shaded region corresponds to a range of one standard deviation of the background.

We searched the SPI-ACS light curve using five timescales from 0.1 to $10 \mathrm{~s}$, within a window of $30 \mathrm{~s}$ before and after the time of GW170817. The local background noise properties are in good agreement with the expectation for the background at the current epoch. On a $100 \mathrm{~ms}$ timescale, we detect only one significant excess with a signal-to-noise ratio $(\mathrm{S} / \mathrm{N})$ of 4.6 , starting at $T_{0, \mathrm{GW}}+1.9 \mathrm{~s}$ (in the geocentric time system; hereafter $T_{0, \mathrm{ACS}}$ ); see Figure 2 . We compute a significance of association between GRB 170817A as observed by INTEGRAL and GW170817 of $3.2 \sigma$. The association significance with the Fermi-GBM observation of GRB 170817A is $4.2 \sigma$ (see Appendix A).

The principal part of the excess gamma-ray emission emerges in just two $50 \mathrm{~ms}$ time bins. The $100 \mathrm{~ms}$ duration firmly places this event in the short GRB class at $>99 \%$ probability (using the GRB duration distribution from Savchenko et al. 2012 and Qin et al. 2013). We should note, however, that the SPI-ACS does not have the capability to observe emission below $\sim 100 \mathrm{keV}$ (due to the limitations of the instrumental low threshold), which might have slightly different temporal characteristics, as reported by Goldstein et al. (2017).

Our coincident observation of the gamma-ray signal permits a substantial improvement of the Fermi-GBM-only localization by exploiting the difference in the gamma-ray arrival times at the location of the two satellites. Using the triangulation annulus reported by Svinkin \& Hurley (2017) we compute that the addition of the INTEGRAL observation reduces the final $90 \%$ GBM localization area by a factor of 1.8 . We refer to the joint LIGO/Virgo, Fermi-GBM, and INTEGRAL/SPI-ACS analysis for more details (LVC et al. 2017). Appendix B summarizes the supporting complete INTEGRAL data set at the time of GRB 170817A.

The majority of sGRBs have a hard spectrum, resulting in a strong detection in the SPI-ACS and/or in IBIS (ISGRI, PICsIT, and/or Veto), as long as the respective instrument is favorably oriented (Savchenko et al. 2012, 2017a). GRB 170817A, on the other hand, was very soft, with most of its energy below $\sim 100 \mathrm{keV}$, apart from a short-hard initial pulse emitting at least up to $200 \mathrm{keV}$ (Goldstein et al. 2017). This results in a reduced SPI-ACS signal significance. We determined that for the location of AT 2017gfo, the SPI-ACS efficiency is smoothly increasing from about $100 \mathrm{keV}$ to $200 \mathrm{keV}$, where it reaches a plateau up to the upper energy threshold of $\sim 80 \mathrm{MeV}$. In Figure 3, we show the region that

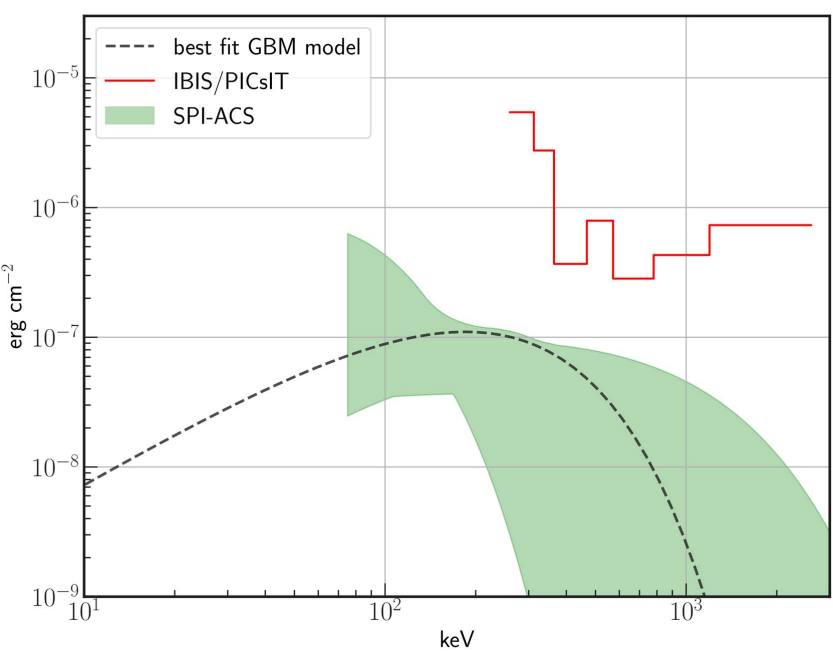

Figure 3. Average hard X-ray/gamma-ray spectrum of the initial pulse of GRB 170817A. The shaded green region corresponds to the range of spectra compatible with the INTEGRAL/SPI-ACS observation (see the text for details). IBIS/PICsIT provides a complementary independent upper limit at high energies; see the text. The best-fit Fermi-GBM model for the spectrum in the same interval (Comptonized model with low-energy index of -0.62 and $E_{\text {peak }}$ of $185 \mathrm{keV}$ ) is shown as a dashed line for comparison (Goldstein et al. 2017).

contains the allowed spectral models consistent with the SPIACS observation. We assume a specific family of models, representative of sGRB spectra not far from the Fermi-GBM best-fit model of GRB 170817A for time time interval $T_{0, \mathrm{GBM}}-0.320-T_{0, \mathrm{GBM}}+0.256-$ (covering the range of spectra consistent with the average or hard peak): Comptonized/cutoff power-law models with $-1.7 \leqslant \alpha \leqslant-0.2$ and $50 \leqslant E_{\text {peak }} \leqslant 300 \mathrm{keV}$. In the same figure, the black dashed line represents the best-fit Fermi-GBM model in the same $0.576 \mathrm{~s}$ long time interval that we used to compare SPI-ACS with the Fermi-GBM results (Goldstein et al. 2017). This comparison nicely displays the consistency of both experiments.

Due to the lack of energy resolution in SPI-ACS, the fluence estimate depends on model assumptions. Using the best-fit Fermi-GBM model (Goldstein et al. 2017) and assuming the time interval $T_{\mathrm{GBM}, 0}-0.320, T_{\mathrm{GBM}, 0}+0.256 \mathrm{~s}$ to match the interval used by Fermi-GBM (Goldstein et al. 2017), we derive a $75-2000 \mathrm{keV}$ fluence of $(1.4 \pm 0.4) \times 10^{-7} \mathrm{erg} \mathrm{cm}^{-2}$ ( $1 \sigma$ error, statistical only). Additionally, the model assumption uncertainty employing the same range of models as used in Figure 3 corresponds to a $75-2000 \mathrm{keV}$ fluence uncertainty of $\pm 0.4 \times 10^{-7} \mathrm{erg} \mathrm{cm}^{-2}$. Possible systematic deviations of the SPI-ACS response, as established by cross-calibration with other gamma-ray instruments (primarily Fermi-GBM and Konus-Wind), corresponds to a further uncertainty of $\pm 0.3 \times$ $10^{-7} \mathrm{erg} \mathrm{cm}^{-2}$.

Due to the limited duration of this event in SPI-ACS, little can be learned directly from the light curve. However, we note that the main prompt component consists of just two bins, with each of the rise time and decay time below $50 \mathrm{~ms}$. Our variability limits are derived for the particularly narrow pulse that characterizes the hardest component of the burst, which is observed by INTEGRAL/SPI-ACS with high effective area. Our results should be compared to the lower-energy morphology probed by Fermi-GBM (see LVC et al. 2017 for details).

After the detection of GRB 170817A, INTEGRAL continued uninterrupted observations of the same sky region until 


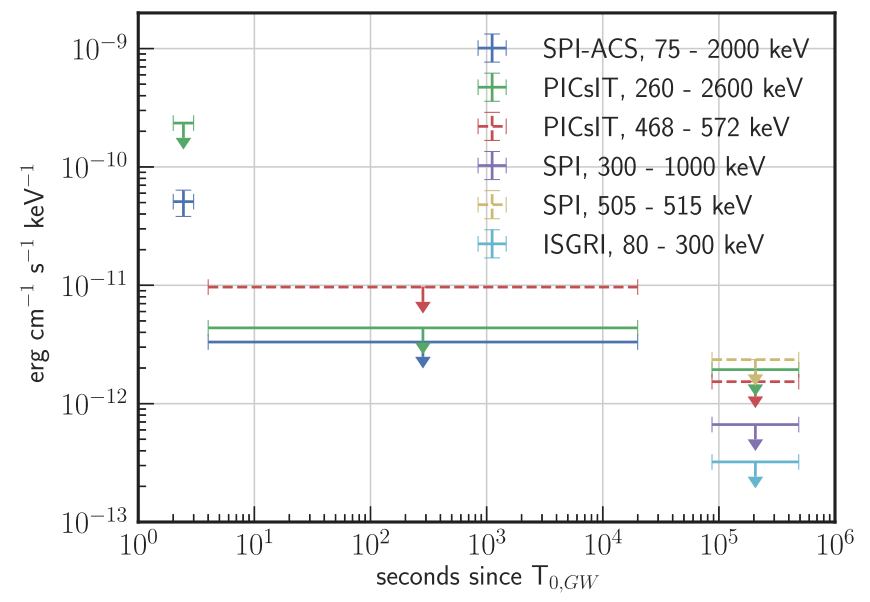

Figure 4. Timeline of the INTEGRAL observations, from the prompt detection with SPI-ACS, through the serendipitous follow-up and toward the targeted follow-up. Dashed lines correspond to the narrowband upper limits. Only selected upper limits are shown; for a complete summary of the observations, see Table 1, Figure 5, and the text.

20:44:01 (UTC on August 17). During this period, no other bursts or steady emission from the direction of the optical counterpart were detected. We report in detail our flux limits in Appendix C, while in Figure 4, we graphically summarize our results.

\section{Targeted INTEGRAL Follow-up Observation}

\subsection{Search for a Soft Gamma-Ray Afterglow}

INTEGRAL allows us to search for an afterglow emission in a broad energy range from $3 \mathrm{keV}$ to $8 \mathrm{MeV}$. This was covered in detail in Savchenko et al. (2017a), where we exploited the serendipitous coverage of part of the LVT151012 localization region within the field of view (FoV) of the INTEGRAL pointed instruments.

To search for a delayed signal, INTEGRAL performed targeted follow-up observations of the LIGO/Virgo candidate BNS merger G298048 (=GW170817). They started $19.5 \mathrm{hr}$ after the event centered the best Fermi-GBM localization (Connaughton 2017). They covered only a negligible fraction of the LIGO/Virgo localization. Therefore, we avoid discussing this initial part of the follow-up.

The main part of the follow-up observations was centered on the candidate optical counterpart, AT 2017gfo (R.A. = 13:09:48.089, decl. $=-23: 22: 53.35$; Coulter et al. 2017). It spanned from 2017 August 18 at 12:45:10 to 2017 August 23 at 03:22:34 (starting about $24 \mathrm{hr}$ after the LIGO/Virgo event), with a maximum on-source time of $326.7 \mathrm{ks}$. AT 2017 gfo was in the highest sensitivity part of IBIS and SPI FoV in each of the dithered single $\sim 40$ minute long individual pointings that make up INTEGRAL observations; it was in the JEM-X FoV (defined as the region where the sensitivity is no less than a factor of 20 from the optimal) for $95 \%$ of the time, and $29 \%$ of the time in the region with sensitivity no worse than a factor of 2 from the optimal.

We investigated the mosaicked images of the complete observation of IBIS/ISGRI, SPI, and JEM-X. We do not detect any X-ray or gamma-ray counterpart to AT 2017gfo in any of the instruments. The $3 \sigma$ broadband upper limits for an average flux of a source at the position of AT 2017 gfo are presented in Figure 5 and Table 1.

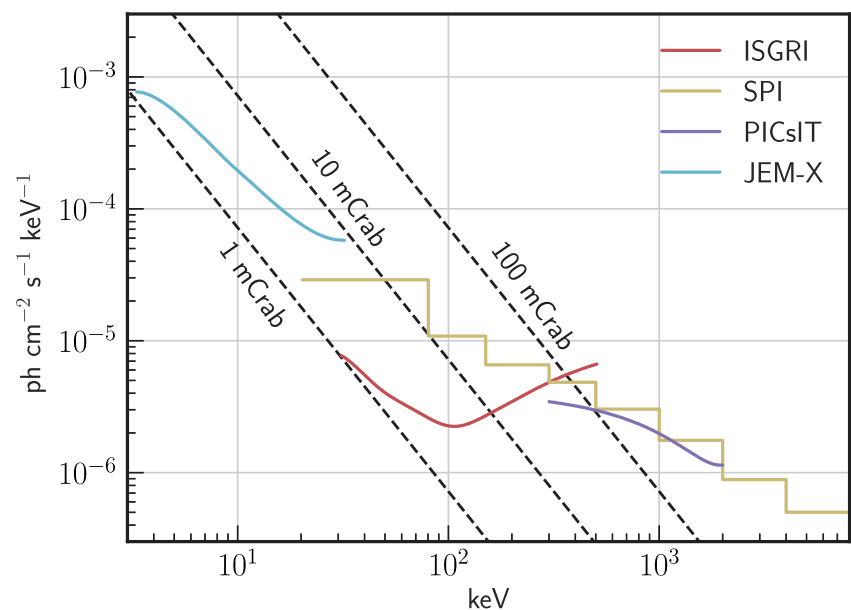

Figure 5. Broadband X-ray to gamma-ray sensitivity reached in the complete INTEGRAL targeted follow-up observation, with a total exposure up to $330 \mathrm{ks}$ (depending on the instrument and the operational mode).

We have also searched for isolated line-like features in IBIS/ ISGRI and SPI data: our preliminary analysis did not identify any such features. In-depth studies will be reported elsewhere. The narrow-line sensitivity reached in the complete follow-up observation is presented in Figure 6.

IBIS, SPI, and JEM-X observed more than $97 \%$ of the LIGO/Virgo localization in the combined observation mosaic. We searched the IBIS/ISGRI, SPI, and JEM-X data for any new point source in the whole $90 \%$ LIGO/Virgo localization region, and we did not find any. The sensitivity depends on the location, with the best value close to that computed for AT 2017gfo. Contours containing regions observed with a sensitivity of at least $50 \%$ and $10 \%$ of the optimal are presented in Figure 7.

\subsection{Search for Optical Emission with the OMC}

The OMC observed the galaxy NGC 4993 including the transient AT 2017gfo from 2017 August 18 at 17:27:59 until 2017 August 22 at 22:56:48 UTC. It was in the OMC FoV for only 29 INTEGRAL pointings (total of $35.7 \mathrm{ks}$ ). Its limited angular resolution and pixel size did not allow us to distinguish between the host galaxy contribution and the transient. The data were analyzed by using the largest OMC photometric aperture $(5 \times 5$ pixels, 90 arcsec diameter $)$ to ensure all of the emission from the host galaxy as well as the transient are included in the aperture. We measured a $\mathrm{V}$ magnitude of $12.67 \pm 0.03$ ( $1 \sigma$ level) for the total emission. No variability was detected in the OMC data at the reported $1 \sigma$ level.

\subsection{Search for Delayed Bursting Activity}

The continuous observation of the AT 2017gfo location performed by INTEGRAL (from 2017 August 18 at 12:45:10 to 2017 August 23 at 03:22:34 UT with a coverage fraction of $80 \%$ ) allows us to also search for any short (magnetar-like) or long bursts from this source. We used IBIS/ISGRI light curves in two energy ranges: $20-80 \mathrm{keV}$ and $80-300 \mathrm{keV}$, on $100 \mathrm{~ms}$, $1 \mathrm{~s}, 10 \mathrm{~s}$, and $100 \mathrm{~s}$ timescales. We did not find any deviations from the background, and set a $3 \sigma$ upper limit on any possible $1 \mathrm{~s}$ long burst flux of $1.0 \mathrm{Crab}\left(1.4 \times 10^{-8} \mathrm{erg} \mathrm{cm}^{-2} \mathrm{~s}^{-1}\right)$ in the $20-80 \mathrm{keV}$, and $6.8 \mathrm{Crab}\left(7.8 \times 10^{-8} \mathrm{erg} \mathrm{cm}^{-2} \mathrm{~s}^{-1}\right)$ in the 
Table 1

Summary of Sensitivities for the Different Instruments On Board INTEGRAL to a Source at the Location of AT 2017gfo

\begin{tabular}{|c|c|c|c|c|c|c|}
\hline \multirow{2}{*}{ Instrument } & \multirow{2}{*}{$\begin{array}{l}\text { Field of View } \\
\left(\mathrm{deg}^{2}\right)\end{array}$} & \multirow{2}{*}{ Angular Resolution } & \multirow{2}{*}{ Energy Range } & \multicolumn{3}{|c|}{$3 \sigma$ Sensitivity } \\
\hline & & & & (mCrab) & $\left(\right.$ erg $\left.\mathrm{cm}^{-2} \mathrm{~s}^{-1}\right)$ & $\left(\mathrm{erg} \mathrm{s}^{-1}\right)$ \\
\hline \multirow[t]{2}{*}{$\overline{J E M-X}$} & 110 & $3^{\prime}$ & $3-10 \mathrm{keV}$ & 1.2 & $1.9 \times 10^{-11}$ & $3.6 \times 10^{42}$ \\
\hline & & & $10-25 \mathrm{keV}$ & 0.64 & $7.0 \times 10^{-12}$ & $1.3 \times 10^{42}$ \\
\hline \multirow[t]{3}{*}{ IBIS/ISGRI } & 823 & $12^{\prime}$ & $20-80 \mathrm{keV}$ & 2.6 & $3.8 \times 10^{-11}$ & $7.3 \times 10^{42}$ \\
\hline & & & $80-300 \mathrm{keV}$ & 6.2 & $7.1 \times 10^{-11}$ & $1.4 \times 10^{43}$ \\
\hline & & & $300-500 \mathrm{keV}$ & 290 & $1.0 \times 10^{-9}$ & $1.9 \times 10^{44}$ \\
\hline \multirow[t]{4}{*}{ IBIS/PICsIT } & 823 & $24^{\prime}$ & $208-468 \mathrm{keV}$ & 36 & $2.1 \times 10^{-10}$ & $4.0 \times 10^{43}$ \\
\hline & & & $468-572 \mathrm{keV}$ & 128 & $1.6 \times 10^{-10}$ & $3.1 \times 10^{43}$ \\
\hline & & & $572-1196 \mathrm{keV}$ & 216 & $8.7 \times 10^{-10}$ & $1.7 \times 10^{44}$ \\
\hline & & & $1196-2600 \mathrm{keV}$ & 973 & $3.3 \times 10^{-9}$ & $6.4 \times 10^{44}$ \\
\hline \multirow[t]{8}{*}{ SPI } & 794 & 2.5 & $20-80 \mathrm{keV}$ & 3.8 & $5.6 \times 10^{-11}$ & $1.1 \times 10^{43}$ \\
\hline & & & $80-150 \mathrm{keV}$ & 16.4 & $9.8 \times 10^{-11}$ & $1.9 \times 10^{43}$ \\
\hline & & & $150-300 \mathrm{keV}$ & 43 & $2.4 \times 10^{-10}$ & $4.6 \times 10^{43}$ \\
\hline & & & $300-500 \mathrm{keV}$ & 135 & $4.7 \times 10^{-10}$ & $9.0 \times 10^{43}$ \\
\hline & & & $500-1000 \mathrm{keV}$ & 308 & $1.2 \times 10^{-9}$ & $2.3 \times 10^{44}$ \\
\hline & & & $1000-2000 \mathrm{keV}$ & 866 & $2.8 \times 10^{-9}$ & $5.4 \times 10^{44}$ \\
\hline & & & $2000-4000 \mathrm{keV}$ & 2117 & $5.7 \times 10^{-9}$ & $1.1 \times 10^{45}$ \\
\hline & & & $4000-8000 \mathrm{keV}$ & 5812 & $1.3 \times 10^{-8}$ & $2.5 \times 10^{45}$ \\
\hline
\end{tabular}

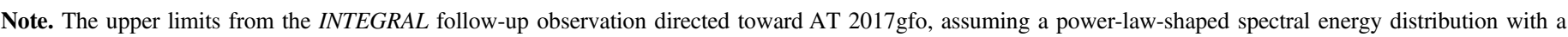

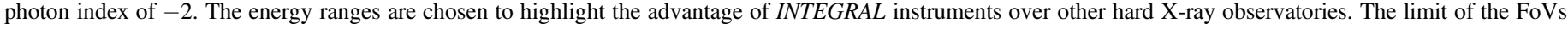
has been set to the point where a worsening of the instrument sensitivity by a factor of 20 compared to the on-axis value is reached.

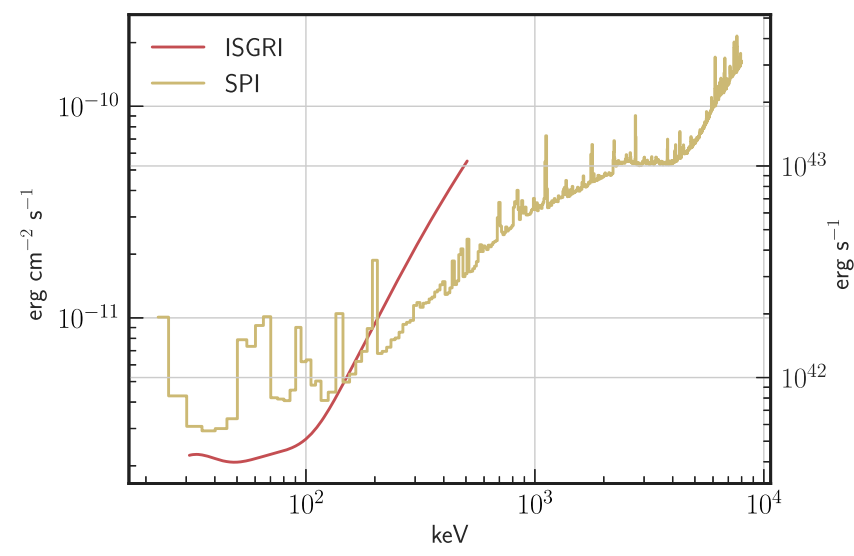

Figure 6. Narrow-line sensitivity in the X-ray/gamma-ray band reached in the complete INTEGRAL targeted follow-up observation, with a total exposure of $330 \mathrm{ks}$ for each of the instruments. The units of the right vertical axis correspond to the luminosity, assuming a distance to the source of $40 \mathrm{Mpc}$.

80-300 keV energy range. The $3 \sigma$ upper limit on a $100 \mathrm{~ms}$ timescale results in $3.0 \mathrm{Crab}\left(4.5 \times 10^{-8} \mathrm{erg} \mathrm{cm}^{-2} \mathrm{~s}^{-1}\right)$ in the $20-80 \mathrm{keV}$, and $21 \mathrm{Crab}\left(2.4 \times 10^{-7} \mathrm{erg} \mathrm{cm}^{-2} \mathrm{~s}^{-1}\right)$ in the $80-300 \mathrm{keV}$ energy range. Assuming a distance of $40 \mathrm{Mpc}$ (see online data of Crook et al. 2007), these limits can be interpreted as constraints on the burst luminosity on a $1 \mathrm{~s}(100 \mathrm{~ms})$ timescale of $2.6 \times 10^{45} \mathrm{erg} \mathrm{s}^{-1}\left(8.5 \times 10^{45} \mathrm{erg} \mathrm{s}^{-1}\right)$ in the $20-80 \mathrm{keV}$ energy range. In the $80-300 \mathrm{keV}$ energy range, the luminosity is constrained to be less than $1.5 \times 10^{46} \mathrm{erg} \mathrm{s}^{-1}$ $\left(4.6 \times 10^{46} \mathrm{erg} \mathrm{s}^{-1}\right)$. Bursts exceeding such luminosities were previously observed from magnetars. SGR 1806-20, for example, produced a giant flare with a total energy of $2 \times 10^{46}$ erg (Hurley et al. 2005), while individual flares from, e.g., 1E 1547.0-5408 exceeded the energy of $10^{46} \mathrm{erg}$ (Mereghetti et al. 2009; Savchenko et al. 2010).

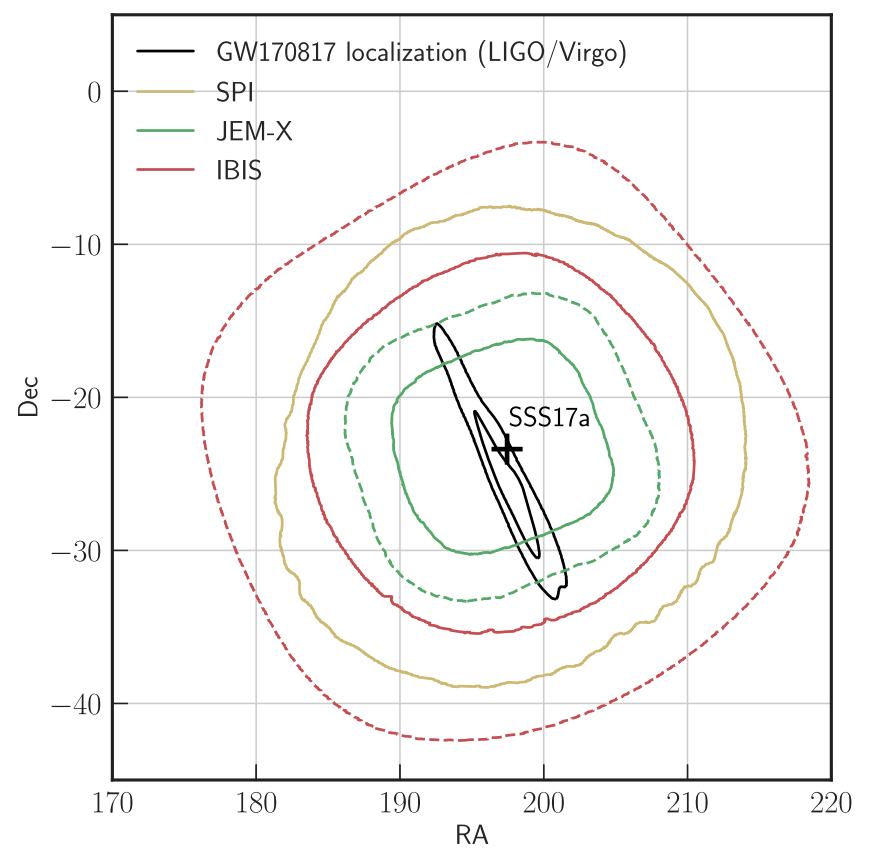

Figure 7. Sensitivity levels (50\%—-solid line and 10\%—-dashed line, of the optimal sensitivity achieved for AT $2017 \mathrm{gfo} / \mathrm{SSS} 17 \mathrm{a}$ ) of the complete IBIS, JEM-X, and SPI mosaics of the targeted INTEGRAL follow-up observation, compared to the most accurate LALInferrence LIGO/Virgo localization of GW170817 (50\% and 90\% confidence containment, black solid lines; LIGO Scientific Collaboration \& Virgo Collaboration 2017c) and the AT 2017gfo location (Coulter et al. 2017).

\section{Discussion}

The detection of GRB 170817A by INTEGRAL and Fermi in unambiguous coincidence with GW170817 is the first definitive proof that at least some sGRBs can be associated with BNS merger events. The duration of the GRB as measured 
by INTEGRAL above $\sim 100 \mathrm{keV}, 100 \mathrm{~ms}$, firmly assigns the GRB 170817A to the short GRB class. LVC et al. (2017) extensively discuss the implications of the joint $\mathrm{GW}$ and gamma-ray observation for the luminosity function and structure of the sGRB population.

Future observations of similar events will be decisive in constraining the properties of the BNS merger counterparts. INTEGRAL exhibits an exceptionally unocculted (>99.9\%) sky view at every moment when it is observing, i.e., for about $85 \%$ of the total mission lifetime. With its high sensitivity above $\sim 100 \mathrm{keV}$, INTEGRAL/SPI-ACS has demonstrated its capability of detecting also fairly weak and soft transients like GRB 170817A. In the future, INTEGRAL will be able to systematically detect counterparts to the GW events or to put tight upper limits on their presence. The advantage of its exceptionally high effective area above $\sim 100 \mathrm{keV}$ will be even more important for the events with harder spectra, which is what is expected for typical sGRBs.

The possibility of forming a (short-lived) magnetar in a BNS merger has been extensively discussed in the past (e.g., Price \& Rosswog 2006; Metzger \& Piro 2014; Giacomazzo et al. 2015; Fernández \& Metzger 2016). It has been suggested that a newborn magnetar is responsible for part of the afterglow emission (e.g., Rowlinson et al. 2013). In principle, it is not clear if a newborn magnetar, formed in the merger, is more likely to produce bursts during the first days after the merger (which are covered by the continuous INTEGRAL observation). This could be reasonably expected because at early times, the magnetic energy is maximal and it rapidly dissipates. Intense $\mathrm{X}$-ray flares could occur associated with frequent reorganization in the magnetic field structure, as well as in connection to a delayed accretion. We have ruled out, however, the existence of strong magnetar-like bursts in our targeted follow-up observation.

Interestingly, the amount of energy released in GRB 170817A (LVC et al. 2017) is similar to that found during the giant flares of magnetars, such as SGR 1806-20 (Hurley et al. 2005). Magnetar flares are associated with long-lived objects while the GRB 170817A was a one-time event of a BNS merger. Nevertheless, the similarity in the most basic observational properties is intriguing and it may point toward a similarity of the physical processes involved.

At late times after the initial gamma-ray burst, the luminosity of kilonovae is largely fueled by radioactive decays of r-process elements released in the coalescence (see, e.g., Metzger 2017 for a review on kilonova mechanism). Under favorable conditions, a forest of nuclear gamma-ray lines produced in these decays may be detectable by a suitable gamma-ray spectrometer such as INTEGRAL/SPI (Hotokezaka et al. 2016). If the lines are broad or appear at low energies $(<100 \mathrm{keV})$, IBIS could also detect them, with a similar significance. We did not find any such emission feature with INTEGRAL/SPI or IBIS, and we set an upper limit as displayed in Figure 6 for narrow lines. However, for sufficiently broad lines, the emission pattern can resemble a nearly continuous spectrum (as discussed in Hotokezaka et al. 2016 for highvelocity ejecta). Thus, the continuum emission upper limit can be applied (see Figure 5 and Table 1).

To the best of our knowledge, the most favorable predictions for a combined decay line flux 1 day after the merger and at the AT $2017 \mathrm{gfo}$ distance are of the order of $3.6 \times 10^{-12}$

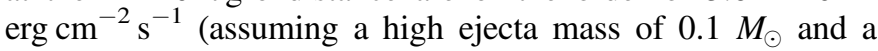

velocity of $0.3 c$; Hotokezaka et al. 2016) in the $300 \mathrm{keV}-1$ $\mathrm{MeV}$ band; this is considerably below our best upper limit in the same energy range, i.e., $1.7 \times 10^{-9} \mathrm{erg} \mathrm{cm}^{-2} \mathrm{~s}^{-1}$.

The detectability of gamma-ray emission resulting from the $\mathrm{e}^{+/-}$annihilation strongly depends on the final photon spectrum, which is in turn determined by the conditions in which annihilation occurs. The final spectrum could in principle include a narrow or a broad, blueshifted or redshifted line-like feature near $511 \mathrm{keV}$, or may be dominated by a very extended excess in the soft gamma-ray energy range (e.g., Svensson 1987; Maciolek-Niedzwiecki et al. 1995). To give a general idea about the sensitivity of INTEGRAL, we consider the IBIS/PICsIT upper limits in the energy range $468-572 \mathrm{keV}$, i.e., around the $511 \mathrm{keV}$ annihilation line, during the targeted follow-up observation. This limit corresponds to $3.1 \times 10^{43} \mathrm{erg} \mathrm{s}^{-1}$ (see Table 1). This luminosity roughly constraints the total rate of annihilation to less than $1.7 \times$ $10^{-13} M_{\odot} \mathrm{s}^{-1}$. A particularly stringent upper limit can be set by SPI on the flux of a narrow annihilation line between 505 and $515 \mathrm{keV}$, which is less than $4.5 \times 10^{42} \mathrm{erg} \mathrm{s}^{-1}$.

\section{Conclusions}

We reported the independent INTEGRAL detection of a sGRB (GRB 170817A), in coincidence with that found by Fermi-GBM (the association significance between INTEGRAL and Fermi-GBM is $4.2 \sigma$ ), which is for the first time unambiguously associated to the GW event GW170817 observed by LIGO/Virgo and consistent with a BNS merger. The significance of association between the independent INTEGRAL GRB detection and GW170817 is $3.2 \sigma$. This is a turning point for multi-messenger astrophysics.

This observation is compatible with the expectation that a large fraction (if not all) BNS mergers might be accompanied by a prompt gamma-ray flash (LVC et al. 2017), detectable by INTEGRAL/SPI-ACS and other facilities. INTEGRAL independently detects more than 20 confirmed sGRBs per year (Savchenko et al. 2012) in a broad range of fluences. With the growing sensitivity of the LIGO and Virgo observatories, being joined in the future by other observatories, we expect to detect more and more short GRBs associated with BNS mergers.

Additionally, we have exploited the unique uninterrupted serendipitous INTEGRAL observations available immediately after GRB 170817A/GW170817 (lasting about $20 \mathrm{ks),} \mathrm{as} \mathrm{well}$ as dedicated targeted follow-up observations carried out by INTEGRAL, starting as soon as $19.5 \mathrm{hr}$ after the GRB/GW (lasting in total 5.1 days). No hard X-ray or gamma-ray signal above the background was found. By taking advantage of the full sensitivity and wide FoV of the combination of the IBIS, SPI, and JEM-X instruments, we provide a stringent upper limit over a broad energy range, from $3 \mathrm{keV}$ up to $8 \mathrm{MeV}$. The INTEGRAL upper limits above $80 \mathrm{keV}$ are tighter than those set by any other instrument and constrain the isotropic-equivalent luminosity of the soft gamma-ray afterglow to less than $1.4 \times 10^{43} \mathrm{erg} \mathrm{s}^{-1}(80-300 \mathrm{keV})$, assuming a distance of $40 \mathrm{Mpc}$ to the source. Our data exclude the possibility that a short- or a long-lasting bright, hard X-ray and/or soft gammaray phase of activity followed GRB 170817A/GW170817.

With these results, we show that INTEGRAL continues to play a key role in the rapidly emerging multi-messenger field by constraining both the prompt and delayed gamma-ray emission associated with compact object mergers. 
This work is based on observations with INTEGRAL, an ESA project with instruments and science data center funded by ESA member states (especially the PI countries: Denmark, France, Germany, Italy, Switzerland, Spain), and with the participation of Russia and the USA. The INTEGRAL SPI project has been completed under the responsibility and leadership of CNES. The SPI-ACS detector system has been provided by MPE Garching/Germany. The SPI team is grateful to ASI, CEA, CNES, DLR, ESA, INTA, NASA, and OSTC for their support. The Italian INTEGRAL team acknowledges the support of ASI/INAF agreement No. 2013-025-R.1. R.D. and A.v.K. acknowledge the German INTEGRAL support through DLR grant 50 OG 1101. A.L. and R.S. acknowledge the support from the Russian Science Foundation (grant 14-2200271). A.D. is funded by Spanish MINECO/FEDER grant ESP2015-65712-C5-1-R. Some of the results in this Letter have been derived using the HEALPix (Górski et al. 2005) package. We are grateful the François Arago Centre at APC for providing computing resources, and VirtualData from LABEX P2IO for enabling access to the StratusLab academic cloud. We acknowledge the continuous support by the INTEGRAL Users Group and the exceptionally efficient support by the teams at ESAC and ESOC for the scheduling of the targeted follow-up observations. We are grateful to the LVC and Fermi-GBM teams for their suggestions on earlier versions of this Letter. Finally, we thank the anonymous referee for constructive suggestions.

\section{Appendix A Association Significance}

It is well known that cosmic-ray (CR) interactions can cause short spikes in the ACS light curve (Savchenko et al. 2012). The false-alarm rate (FAR) in SPI-ACS for short and weak events, like GRB 170817A, is largely determined by the ability to discriminate between CR-induced and astrophysical events. Following Savchenko et al. (2012), we exploit the universality of the CR-induced spike temporal profile in SPI-ACS and compute the significance of an event to adhere to the NULL hypothesis of having a spike-like profile; we then multiply this number by the $\mathrm{S} / \mathrm{N}$ of the event to obtain a numeric ranking.

We used data from the same INTEGRAL spacecraft revolution (2017 August 15 at 15:27:42-2017 August 17 at 18:26:32 UTC, $51 \mathrm{hr}$ in total) to compute the FAR for the events with a rank not smaller than that of GRB 170817A. This results in $2.2 \times 10^{-5} \mathrm{~Hz}$ and can be used to compute a post-trial false-alarm probability for an excess at $T_{0, \mathrm{ACS}}$ to be associated with the $\mathrm{GW}$ trigger as $P=2 \times 2.2 \times 10^{-5} \mathrm{~Hz} \times 2 \mathrm{~s} \times(1+\log (30 \mathrm{~s} / 0.1 \mathrm{~s}) \sim 0.07 \%$ $(3.2 \sigma)$. Here, we use the $2 \mathrm{~s}$ time difference with the association target (GW170817), the $30 \mathrm{~s}$ half time window of the search, and the $0.1 \mathrm{~s}$ for the phase step in the minimal search timescale. The factor of two is due to counting both before and after the trigger.

The evidence for association with the Fermi-GBM GRB detection can be derived by assuming an association time difference of $50 \mathrm{~ms}$. This is a conservative value taking into account the time bin of SPI-ACS and a marginally allowed offset between the temporal profiles of two events, with the light travel time correction assuming the location of AT 2017 gfo. This results in an association significance of $4.2 \sigma$, providing strong evidence that the event detected by INTEGRAL/SPI-ACS is associated with the GRB 170817A, detected by the Fermi-GBM onboard algorithms. It is interesting to derive an association significance using only the Fermi-GBM and INTEGRAL/SPI-ACS results, i.e., using the GBM location of GRB 170817A for the light travel time correction. The extent of the localization region derived from the GBM data alone accounts for an additional time uncertainty of $100 \mathrm{~ms}$, resulting in an association significance of $3.9 \sigma$.

\section{Appendix B \\ Search for Prompt Emission with IBIS and SPI}

We have inspected data of the IBIS/ISGRI, IBIS/PICsIT, and SPI main detectors and did not find any excess at the

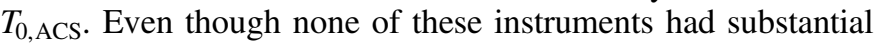
sensitivity in the direction of AT 2017gfo (see Savchenko et al. $2017 \mathrm{a}$ for the all-sky angular dependency of sensitivity of different instruments), this inspection is particularly interesting because particle background variations tend to appear in multiple instruments at once. The non-observation of any excess in instruments where we do not expect an astrophysical signal disfavors the hypothesis that local particle background excess contributed to the event and further supports that our detection is associated with AT 2017 gfo and the GW event. Note that we did not use the X-ray information from the JEM$\mathrm{X}$ instrument, since in its energy range $(<30 \mathrm{keV})$ it was heavily shielded from any emission in the direction of AT 2017gfo.

\section{Appendix C \\ Search for an Early Soft Gamma-Ray Afterglow}

After the detection of GRB 170817A, INTEGRAL continued to point toward the same sky region (i.e., essentially with no change to the response for the position of AT 2017gfo) in stable background conditions, until the instruments were switched off for the perigee passage at 20:44:01 (UTC on August 17). The total duration of this observation was about $20 \mathrm{ks}$. Exploiting the all-sky sensitivity of SPI-ACS and IBIS/ PICsIT, we derive upper limits on any early gamma-ray afterglow for this period of time.

Using the background rate from the earlier part of the INTEGRAL spacecraft revolution 1851 (the revolution that contains the GRB 170817A) and the data of neighboring revolutions, we estimate a $3 \sigma$ upper limit on the average flux of any new source in the single broad energy range 75-2000 keV accessible to SPI-ACS of $280 \mathrm{mCrab}$ or $1.2 \times 10^{45} \mathrm{erg} \mathrm{s}^{-1}$, assuming a Crab-like spectrum.

While the response of IBIS/PICsIT to a GRB-like spectrum in the direction of AT 2017gfo was less than optimal, the energy resolution in the PICsIT spectral-timing mode allows us to set a constraining upper limit on emission limited to a narrow range of gamma-ray energies. The $3 \sigma$ upper limits on a source flux average over the studied time interval exploiting PICsIT have been determined in three energy bands. Two broad ones and a narrow one centered on $511 \mathrm{keV}$ to search for broad line emission. The upper limits from these serendipitous observations are: $2.8 \times 10^{45} \mathrm{erg} \mathrm{s}^{-1}(260-468 \mathrm{keV}), 3.1 \times 10^{44} \mathrm{erg} \mathrm{s}^{-1}$ $(468-572 \mathrm{keV})$, and $3.3 \times 10^{45} \mathrm{erg} \mathrm{s}^{-1}(572-2600 \mathrm{keV})$.

Using the SPI-ACS data in the same time interval, we also searched for isolated short bursts on timescales from $100 \mathrm{~ms}$ to $10 \mathrm{~s}$. We do not find any evidence for further bursting activity and set an upper limit on any excess at the level of $\sim 5 \times 10^{-7} \mathrm{erg} \mathrm{cm}^{-2}$ on $1 \mathrm{~s}$ timescale for the $75 \mathrm{keV}-2 \mathrm{MeV}$ energy range. 


\section{ORCID iDs}

V. Savchenko (1) https://orcid.org/0000-0001-6353-0808

C. Ferrigno (1) https://orcid.org/0000-0003-1429-1059

A. Bazzano (ib https://orcid.org/0000-0002-2017-4396

E. Bozzo (1) https://orcid.org/0000-0002-7504-7423

J. Chenevez (1) https://orcid.org/0000-0002-4397-8370

T. J.-L. Courvoisier (10 https://orcid.org/0000-0003-2396-6249

L. Hanlon (i) https://orcid.org/0000-0003-2931-3732

E. Jourdain (1) https://orcid.org/0000-0001-9932-3288

A. von Kienlin (1) https://orcid.org/0000-0002-0221-5916

P. Laurent (1) https://orcid.org/0000-0001-9094-0335

A. Lutovinov (i) https://orcid.org/0000-0002-6255-9972

S. Mereghetti i https://orcid.org/0000-0003-3259-7801

\section{References}

Abbott, B. P., Abbott, R., Abbott, T. D., et al. 2016a, PhRvL, 116, 061102 Abbott, B. P., Abbott, R., Abbott, T. D., et al. 2016b, PhRvL, 116, 241103 Abbott, B. P., Abbott, R., Abbott, T. D., et al. 2016c, LRR, 19, 1

Abbott, B. P., Abbott, R., Abbott, T. D., et al. 2017a, PhRvL, 118, 221101

Abbott, B. P., Abbott, R., Abbott, T. D., et al. 2017b, PhRvL, https://doi.org/ 10.1103/PhysRevLett.119.161101

Acernese, F., Agathos, M., Agatsuma, K., et al. 2015, CQGra, 32, 024001

Berger, E. 2014, ARA\&A, 52, 43

Blinnikov, S. I., Novikov, I. D., Perevodchikova, T. V., \& Polnarev, A. G. 1984, SvAL, 10, 177

Connaughton, V. 2017, GCN, 21505

Coulter, D. A., Kilpatrick, C. D., Siebert, M. R., et al. 2017, GCN, 21529

Crook, A. C., Huchra, J. P., Martimbeau, N., et al. 2007, ApJ, 655, 790

D'Avanzo, P. 2015, JHEAp, 7, 73

D’Elia, V., Pian, E., Piranomonte, S., et al. 2017, GCN, 21592

DESGW+Community Team 2017, GCN, 21530

Evans, P. A., Kennea, J. A., Breeveld, A. A., et al. 2017, GCN, 21550

Fernández, R., \& Metzger, B. D. 2016, ARNPS, 66, 23

Foley, R. 2017, GCN, 21557

Gehrels, N., \& Meszaros, P. 2012, Sci, 337, 932

Giacomazzo, B., Zrake, J., Duffell, P. C., MacFadyen, A. I., \& Perna, R. 2015, ApJ, 809, 39

Goldstein, A., Veres, P., Burns, E., et al. 2017, ApJL, https://doi.org/10.3847/ 2041-8213/aa8f41

Górski, K. M., Hivon, E., Banday, A. J., et al. 2005, ApJ, 622, 759

Hotokezaka, K., Wanajo, S., Tanaka, M., et al. 2016, MNRAS, 459, 35

Hurley, K., Boggs, S. E., Smith, D. M., et al. 2005, Natur, 434, 1098
Labanti, C., Di Cocco, G., Ferro, G., et al. 2003, A\&A, 411, L149

Lebrun, F., Leray, J. P., Lavocat, P., et al. 2003, A\&A, 411, L141

LIGO Scientific Collaboration, Aasi, J., Abbott, B. P., et al. 2015, CQGra, 32, 074001

LIGO Scientific Collaboration \& Virgo Collaboration 2017a, GCN, 21505

LIGO Scientific Collaboration \& Virgo Collaboration 2017b, GCN, 21513

LIGO Scientific Collaboration \& Virgo Collaboration 2017c, GCN, 21527

LIGO Scientific Collaboration \& Virgo Collaboration 2017d, GCN, 21509

LIGO Scientific Collaboration \& Virgo Collaboration 2017e, GCN, 21474

Lund, N., Budtz-Jørgensen, C., Westergaard, N. J., et al. 2003, A\&A, 411, L231

LVC, FermiGBM, INTEGRAL et al. 2017, ApJL

Lyman, J., Homan, S., Maguire, K., et al. 2017, GCN, 21582

Maciolek-Niedzwiecki, A., Zdziarski, A. A., \& Coppi, P. S. 1995, MNRAS, 276, 273

Mas-Hesse, J. M., Giménez, A., Culhane, J. L., et al. 2003, A\&A, 411, L261

Mereghetti, S., Götz, D., Weidenspointner, G., et al. 2009, ApJL, 696, L74

Metzger, B. D. 2017, LRR, 20, 3

Metzger, B. D., \& Piro, A. L. 2014, MNRAS, 439, 3916

Nakar, E. 2007, PhR, 442, 166

Price, D. J., \& Rosswog, S. 2006, Sci, 312, 719

Qin, Y., Liang, E.-W., Liang, Y.-F., et al. 2013, ApJ, 763, 15

Quadrini, E. M., Bazzano, A., Bird, A. J., et al. 2003, A\&A, 411, L153

Rowlinson, A., O'Brien, P. T., Metzger, B. D., Tanvir, N. R., \& Levan, A. J. 2013, MNRAS, 430, 1061

Savchenko, V., Bazzano, A., Bozzo, E., et al. 2017a, A\&A, 603, A46

Savchenko, V., Bazzano, A., Bozzo, E., et al. 2017b, GCN, 21507

Savchenko, V., Neronov, A., Beckmann, V., Produit, N., \& Walter, R. 2010, A\&A, 510, A77

Savchenko, V., Neronov, A., \& Courvoisier, T. J.-L. 2012, A\&A, 541, A122

Siebert, M. R., Foley, R. J., Drout, M. R., et al. 2017, ApJL, https://doi.org/ 10.3847/2041-8213/aa905e

Soares-Santos, M., Holz, D. E., Annis, J., et al. 2017, ApJL, https://doi.org/ 10.3847/2041-8213/aa9059

Svensson, R. 1987, MNRAS, 227, 403

Svinkin, D., Hurley, K. \& on behalf of the IPN 2017, GCN, 21515

Tanaka, M., Kato, D., Gaigalas, G., et al. 2017, ApJ, submitted (arXiv:1708. 09101)

Troja, E., Piro, L., van Eerten, A., et al. 2017a, Natur, https://doi.org/10.1038/ nature 24290

Troja, E., Watson, A., Covino, S., et al. 2017b, GCN, 21682

Ubertini, P., Lebrun, F., di Cocco, G., et al. 2003, A\&A, 411, L131

Valenti, S., Sand, D. J., Yang, S., et al. 2017, ApJL, https://doi.org/10.3847/ 2041-8213/aa8edf

Vedrenne, G., Roques, J.-P., Schönfelder, V., et al. 2003, A\&A, 411, L63

von Kienlin, A., Beckmann, V., Rau, A., et al. 2003, A\&A, 411, L299

von Kienlin, A., Meegan, C., Goldstein, A., et al. 2017, GCN, 21520

Winkler, C., Courvoisier, T. J.-L., Di Cocco, G., et al. 2003, A\&A, 411, L1

Yang, S., Valenti, S., Sand, D., et al. 2017, GCN, 21531 